\title{
The Electronic Structure of Disordered Alloys*
}

\author{
J. L. Beeby \\ Theoretical Physics Division, Atomic Energy Research Establishment, Harwell, Didcot, Berks., England
}

(October 10, 1969)

\begin{abstract}
The problem of calculating the electronic density of states in an alloy is considered from first principles. Choosing a suitably simplified model potential a diagrammatic expansion is discussed within which the various existing theories can be compared. Some comments are made on the comparison with experiment.
\end{abstract}

Key words: Density of states; disordered alloys; one-electron propagator; perturbation expansion; sum rule.

\section{Introduction}

It has long been appreciated that the information obtainable from experiments on alloys provides a useful supplement to one's knowledge of the pure materials. Much of this information, such as the Hume-Rothery rules, was obtained using binary alloys with similar non-transition-metal constituents. In such an alloy the electron mean free path is long and the alloy can often be regarded as homogeneous. The problem which will be discussed in this paper concerns how to calculate the properties of an alloy in which the constituents are very different. For such an alloy there arises, besides the routine difficulty of choosing an appropriate potential, a major problem involving the order. Perfectly ordered alloys can be handled just as for pure materials, but disordered alloys with strong scattering potentials have needed the development of new theoretical techniques. These methods will be discussed below via a diagrammatic expansion which appears to give improved insight into the whole problem of disorder. No attempt will be made to formally review the literature and no claim is made to completeness. Rather, it is hoped, readers will be better able to judge for themselves the contents of papers in the field.

The paper will begin with a derivation of the alloy potential. The intention here is to clarify, for those not already familiar with the field, what disorder is and how the theorist can describe it by an averaging process.

*An invited paper presented at the 3d Materials Research Symposium, Electronic Density of States, November 3-6, 1969, Gaithersburg, Md.
While concepts of this type are already common, as in statistical mechanics for example, it is clear from the current literature that many authors still misunderstand them in the alloy context. The derivation of the potential is then completed by briefly listing the assumptions required with a few comments on their validity. The potential used is of muffin-tin type with invariant potentials for each constituent. The brevity of this section should not be regarded as indicative of the trivial nature of obtaining the potential, which in itself forms a most interesting and difficult task. The heart of the paper is contained in sections 4-6 where the density of states is obtained by considering the imaginary part of the T-matrix for the alloy. The T-matrix can be expanded in a series involving the individual scattering centers (the muffin tins) in the usual way. The procedure adopted in section 5 concerns the way in which the series is to be averaged term by term. Each term requires the knowledge of a probability function and it is these probability functions which must be approximated if the series is to be resumed. A diagrammatic expansion is given which, it is argued, formally converges like $\mathrm{Z}^{-1}$ where $\mathrm{Z}$ is the number of nearest neighbors. In practice, however, it is the degree of fluctuation which determines the convergence which is anyhow at best asymptotic. Section 6 illustrates these remarks by looking at numerical solutions for different approximations. In the final sections a few examples will be cited of the interrelation between this work and experiment. These are mainly concerned with the transition metals to which the formalism is most appropriate. 


\section{The Meaning of Disorder}

When an alloy sample is made there is much information which is available in principle yet is not available in practice. It is normally possible to determine the content of each constituent, the structure and certain ordering parameters. But it is plainly absurd to expect to know the actual positions of all the atoms in a disordered sample. Thus while an experiment is performed on a particular sample whose atomic positions may be regarded as fixed during the experiment, a theoretical calculation for this sample must proceed in ignorance of the actual positions. The formal device used to off set this ignorance consists of an averaging procedure about which one might make the following comments.

(i) It is expected that all macroscopically identically produced samples will have (within experimental error) identical properties. Systems with large fluctuations in their properties due to unavoidable variations in production need a different approach. Such fluctuations are usually due to variations in some macroscopic parameter not yet controlled in the production process.

(ii) The detailed microscopic order is therefore only important to the extent that certain macroscopic properties (e.g., order parameters) are satisfied.

(iii) Naturally the theoretician is, in these circumstances, at liberty to choose any one microscopic distribution which satisfies the macroscopic restraints. However, since all such distributions are equivalent, it is easier to average over them with a probability function specifying the chance that they occur.

(iv) Such an approach is well known in Statistical Mechanics and works for the same reason: the number of particles involved in the average is very large.

It is most important that any such averaging is made only over observable properties of the sample. As an example consider the density of states which for a given sample might be written $\mathrm{n}\left(\mathrm{E}, \mathrm{q}_{1}, \mathrm{q}_{2}, \ldots \mathrm{q}_{n}\right)$ depending on certain parameters of the sample. If these parameters have a probability of occurence $\mathrm{P}\left(\mathrm{q}_{1} \ldots \mathrm{q}_{n}\right)$ within the constraints of the sample production then the average density of states is $\int \mathrm{n}\left(\mathrm{E}, \mathrm{q}_{1} \ldots \mathrm{q}_{n}\right) \mathrm{P}\left(\mathrm{q}_{1} \ldots \mathrm{q}_{n}\right) \mathrm{dq}_{1} \ldots$ $\mathrm{dq}_{n}$. If the job has been done properly this average value should differ from a typical single alloy value only to the order $(1 / \mathrm{N})$ where $\mathrm{N}$ is the number of atoms.

Averaging in this way makes things a little simpler algebraically because, like the experimenter, the theorist can ignore all except the macroscopic features of the sample. There are some conceptual difficulties, however, which are worth briefly illustrating. Take first a perfect lattice of one type of atom. Figure la shows for a finite number of atoms the energy levels of the system and the singularities which will occur in the complex Eplane for a single particle Green function. A pole will correspond to each energy level. When $\mathrm{N}$ becomes infinite the energy levels pack together and can be described by a density of states $n(E)$ as in figure $1 b$. At
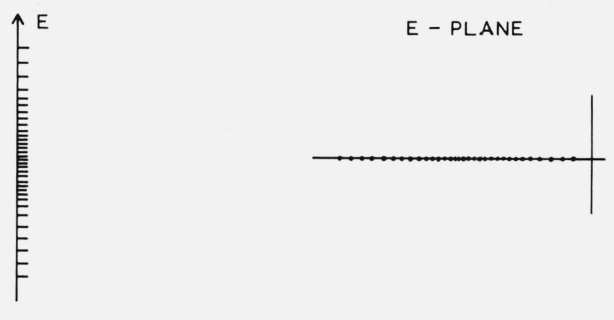

a) N FINITE

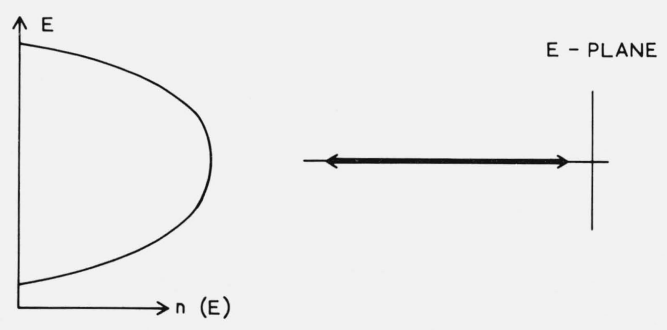

b) N INFINITE

FIGURE 1. The energy levels and complex plane singularities for (a) a finite, (b) an infinite ordered system.

the same time the poles merge together to form a branch cut along a portion of the real axis. For an imperfect arrangement the finite system again has poles on the real axis which will turn into branch cuts upon averaging. When approximations are made in the averaging these branch cuts may have been replaced by poles off the real axis; the importance of remembering that this is a consequence of the approximations has been particularly stressed by S. F. Edwards. A related point concerns the limit $\mathrm{N} \rightarrow \infty$. In the perfect lattice case periodic boundary conditions can be used and no difficulty arises. In the disordered case the limit can only be taken after the averaging procedure. To see this consider a group of $\mathrm{N}$ atoms which is infinitely extended by repetition. The resultant crystal then has $\mathrm{N}$ atoms/unit cell and this is well known to yield a band structure with $\mathrm{N}-1$ band gaps in general. As $\mathrm{N}$ is increased the band gaps become more numerous and narrower, showing that this is not a sensible treatment. 


\section{The Alloy Potential}

A correct procedure for obtaining an alloy potential would be as follows.

(i) The positions of the nuclei and core electrons are considered known. (But will in practice be supposed to lie on a perfect lattice).

(ii) The potential throughout the entire lattice is guessed.

(iii) The density of electrons in the system is then obtained.

(iv) A new estimate of the potential is made.

Such an "in principle" self-consistent calculation must be made for a single unaveraged alloy and is plainly an impossible task. Some part of this self-consistency can be achieved in special cases, e.g., where the Friedel sum rule can be used in dilute alloys. Step (iii) to (iv) is, of course, similar to the same step in the perfect lattice except for the lack of order, but even in the perfect lattice case is far from easy to carry out properly. The novelty of alloy theory lies in steps (ii) to (iii), describing the electron density once the potential is known. Since this step is the primary interest of this paper the discussion can be greatly clarified by choosing a suitably simple form for the potential. Only the one-electron approximation will be considered.

For a dilute alloy the best potential to use is the perfect lattice (host) potential with an additional potential at each impurity site representing the difference between the impurity and host potentials. This potential can then be used in perturbation theory or even in more sophisticated schemes. A modified version of this approach will also work quite well for concentrated nearly-free-electron alloys.

For the case where at least one constituent of the alloy has a strong scattering potential with just-bound or nearly-bound states the muffin-tin approximation is best. The muffin-tin assumption is worse in an alloy than in a pure material because the local interstitial energy may vary from place to place through the alloy. It is hard to see how one can readily estimate the effects of such variation. It is customary to make the additional assumption that the muffin-tin potentials for each constituent are independent of the environment. This is not generally correct; there is a spill over of the impurity potential onto neighboring sites. Unfortunately neglecting this change is inevitable at this stage in the development of the theory and, it will be noticed, resembles neglect of positional relaxation about the impurity site in defect calculations. It should be borne in mind, however, that the level of the theory at which such effects would enter is well beyond anything that will be discussed in this paper. Since it is clear that particular features of the band structure may depend critically upon such local effects, the most obvious example being bound states localized near an impurity atom, the defect type theories plainly have important applications here.

The virtue of the nonoverlapping muffin-tin form of the potential is that in the potential free region between the spheres electrons move as in free space. The motion of an electron can thus be seen as a series of scatterings from individual sites with free electron propagation in between. It is this separation of the scattering events which is so important to what follows.

If the alloy constituents are at sites $R_{i}, \mathrm{i}=1, \ldots \mathrm{N}$ then the potential just discussed may be written for a particular alloy

$$
V(\mathbf{r})=\sum_{i} \nu_{\mu_{i}}\left(\mathbf{r}-\mathbf{R}_{v}\right)
$$

where $\mu_{i}$ is the type of atom at site $R_{i}$ and $v_{\mu}(r)$ is the potential of the $\mu$ 'th atom type. The nonoverlapping restriction is that

$$
\nu_{\mu}(\mathbf{r})=0 \quad r>r_{o}
$$

where $\mathrm{r}_{0}$ is half the near neighbor distance. There is actually no restriction that $v(r)$ be spherically symmetric though this is often quoted as necessary. With this greatly simplified form for the potential it is possible to proceed with the formal theory of disordered alloys.

\section{The T-Matrix}

In the discussion of the density of states in an alloy it is necessary to appeal to the concept of a T-matrix and it is therefore worthwhile to demonstrate that it is actually a simple concept. Indeed, while much of the algebra is rather involved, the use of the T-matrix allows almost classical mental pictures to be used and the algebra very largely suppressed.

Consider then a single scattering center of potential $\mathrm{V}(r)$ with particles incident upon it. It is well known that this problem can be solved in integral equation form as

$$
\psi(\mathbf{r})=\phi(\mathbf{r})-\frac{1}{4 \pi} \int \frac{e^{i k\left|\mathbf{r}-\mathbf{r}^{\prime}\right|}}{\left|\mathbf{r}-\mathbf{r}^{\prime}\right|} V\left(\mathbf{r}^{\prime}\right) \psi\left(\mathbf{r}^{\prime}\right) d \mathbf{r}^{\prime}
$$

where $\phi(r)$ is the incident beam and the second term gives the wave scattered from the potential. Note that the complete wave function $\psi\left(r^{\prime}\right)$ appears in this latter term; if $\phi(r)$ were inserted here the scattering would be given in Born approximation. The T-matrix is formally defined by

$$
V\left(r^{\prime}\right) \psi\left(\mathbf{r}^{\prime}\right)=\int T\left(\mathbf{r}^{\prime}, \mathbf{r}^{\prime \prime}\right) \phi\left(\mathbf{r}^{\prime \prime}\right) d \mathbf{r}^{\prime \prime}
$$


but is best understood in terms of the physical description. The point is that it is very convenient to describe a potential by the scattering it induces among a set of states defined outside the potential and this is what the T-matrix describes. In the crystal these states will be spherical harmonics centered on the atomic position in question. The T-matrix for the potential describes the scattering from one (ingoing) spherical state to another (outgoing) such state.

The infinite crystal potential leads to a T-matrix of different interest. The feature dominant here is that the scattering cross section is infinite for electrons incident on a potential well at an energy at which that well has a bound state. Using this in reverse one may look for the energy levels of the crystal by seeking the poles (or branch cuts) of the T-matrix. Actually the density of states is directly proportional to the imaginary part of the T-matrix and this is the link which will be adopted below. One can now utilize the fact that the total alloy potential is made up of individual scattering sites with their own T-matrices $t_{i}$. All scattering processes from the alloy can be described in terms of the sequence of scatterings from these sites. Thus

$$
T=\sum_{i} t_{i}+\sum_{i \neq j} t_{i} G_{i j} t_{j}+\ldots
$$

where the nth term describes those scatterings from the alloy in which $n$ individual site scatterings occur. $G_{i j}$ describes formally the way in which the electron moves between the scatterings at $\mathrm{i}$ and $\mathrm{j}$ and it is to be noted that two consecutive scatterings cannot be from the same site.

\section{The Alloy Formalism}

The aim of this section is to introduce a diagrammatic technique which carries considerable insight into the nature of the disorder problem. The algebra required to set up the diagrams is given mainly because of its intrinsic interest. This algebra may otherwise largely be skipped since the meaning of the diagrams is fairly readily understood.

For a single alloy the series (1) can be cast in an entirely real form and the imaginary part corresponding to the density of states $\left(\mathrm{n}(\mathrm{E}) \sim \sum \delta\left(\mathrm{E}-\mathrm{E}_{i}\right)\right.$ for this alloy) will only emerge after the infinite series has been summed. In other words the bound states occur at the points where the series diverges. Since the averaging plainly cannot be carried out on the summed T-matrix it is necessary to resort to a term by term averaging followed by the infinite summation. To be specific consider a binary alloy specified by writing at the site $\mathrm{i}$

$$
t_{i}=\sum_{\nu=\alpha, \beta} t_{\nu} c_{i}^{\nu}
$$

where $t_{\alpha}, t_{\beta}$ are the $t$-matrices for potential wells of type $\mathrm{A}$ and $\mathrm{B}$ respectively. Also the $\mathrm{c}_{i}^{\nu}$ are defined by

$$
\begin{aligned}
c_{i}^{\alpha} & =1 & & \text { if the atom at site } i \text { is of type A } \\
& =0 & & \text { otherwise } \\
c_{i}^{\beta} & =1-c_{i}^{\alpha}=1 & & \text { if the atom at site } i \text { is of type B } \\
& =0 & & \text { otherwise }
\end{aligned}
$$

In the average system these $\mathrm{c}_{i}^{\nu}$ are given by certain distribution functions appropriate to the alloy composition in question. It is convenient to introduce dummy variables $c^{\nu}, c^{\nu}=0,1$ so that $c_{\mathrm{i}}^{\nu}=\sum c^{\nu} \delta_{c^{\nu}}, c_{\eta}^{\nu}$. Then the $\mathrm{T}$ matrix becomes

$$
T=\sum_{i, c^{\nu} \nu} t_{\nu} c^{\nu} \delta_{c^{\nu}, c_{i}^{\nu}}+\sum_{\substack{i \neq j \\ c^{\nu}, c^{\mu} \\ \nu, \mu}} t_{\mu} c^{\mu} G_{i j} t_{\nu} c^{\nu} \delta_{c^{\mu}, c_{i}^{\mu}} \delta_{c^{\nu}, c_{j}^{\nu}}+\ldots
$$

and the averaging which must be performed has been entirely concentrated into the Kronecker-delta functions.

Consider these averages. What one requires to know are probability distributions such as

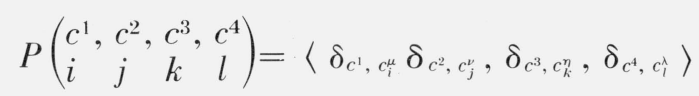

For example, take a completely disordered binary system in which the concentration of the constituent $\mathrm{A}$ is c. Then the probability distribution of the $\mathrm{c}_{i}^{\nu}$ 's is

$$
\underset{\substack{\text { all } \\ \text { sites }}}{\prod}\left\{c \delta_{c_{i}^{\mu}, c_{i}^{a}}+(1-c) \delta_{c_{i}^{\mu}, c_{i}^{\beta}}\right\}
$$

Since the probability of finding atom A is independently c on every site. When this probability function is used to evaluate the average on the right-hand side of (3) the factor in braces in (4) only occurs once for each independent site in (3). This means there is not necessarily one such factor for each Kronecker delta in (3) but rather only one for each independent site. Thus one obtains

$$
\begin{aligned}
& P\left(\begin{array}{llll}
c^{1}, & c^{2}, & c^{3}, & c^{4} \\
i & j & k & l
\end{array}\right)=\sum_{c_{i}^{\mu}, c_{j}^{\mu} \text { etc. }} \Pi^{\prime}\left\{c \delta_{c_{i}^{\mu}, c_{i}^{\alpha}}+(1-c) \delta_{c_{i}^{\mu}, c_{i}^{\beta}}\right\} \\
& \delta_{c^{1}, c_{i}^{\mu}} \ldots \delta_{c, c_{i}^{\lambda}}
\end{aligned}
$$

where the prime on the product denotes the restriction that only independent sites among the $\mathrm{i}, \mathrm{j}, \mathrm{k}, \mathrm{l}$ are included. It is this restriction which is at the heart of the disordered alloy problem. It states that when the same atom appears more than once in a term of the series (2) the average cannot be taken as though each appearance is independent of the others. It is to be 
emphasized that this restriction is geometrical; it is a sort of counting problem. Obviously the magnitude of any counting error will depend on the relative size of the various terms and the precise determination of the physical parameters determining these sizes is the essential problem to be faced. The partial progress in this direction will later be illustrated by example but much remains to be done.

A common first step in dealing with a restriction is to first ignore it and then make successively more accurate corrections. This procedure is readily adopted here. Consider the product over independent sites of some factor $\mathrm{f}_{i}$ :

$$
\begin{gathered}
\underset{\substack{\text { indep. } \\
\text { sites }}}{\Pi} f_{i}=\prod_{\substack{\text { indep. } \\
\text { sites }}} f_{i} \Pi\left[\begin{array}{c}
\text { Other } \\
\text { sites }
\end{array}\right. \\
=\prod_{\substack{\text { all } \\
\text { sites }}} f_{i}\left\{1+\sum_{\substack{\text { other } \\
\text { sites }}} \frac{\left(1-f_{i}\right)}{f_{i}}+\sum_{\substack{\text { two other } \\
\text { sites }}}\right. \\
\left.\quad \frac{\left(1-f_{i}\right)}{f_{i}} \frac{\left(1-f_{j}\right)}{f_{j}}+\ldots\right\}
\end{gathered}
$$

where the last line is obtained by expanding the second product. Clearly the correct result will only be regained after all the terms in the sum have been included. This formula allows a simple diagrammatic representation in which all the sites appearing in a term are given as dots on a line as in figure $2 \mathrm{a}$. The dots themselves represent the $t$-matrices and the lines between the dots represent the propagators $\mathrm{G}_{i j}$. In this series of scatterings the repetition of a given scattering center can be treated by the device of eq (6). The first term in the sum in (6) corresponds to ignoring the restriction in which case the probability distribution will be given by

$$
P\left(\begin{array}{l}
c^{1} c^{2} c^{3} \\
i, j, k, \ldots
\end{array}\right) \simeq P\left(\begin{array}{c}
c^{1} \\
i
\end{array}\right) P\left(\begin{array}{c}
c^{2} \\
j
\end{array}\right) P\left(\begin{array}{c}
c^{3} \\
k
\end{array}\right) \ldots
$$

where

$$
P\left(\begin{array}{l}
c^{1} \\
i
\end{array}\right)=\sum_{c_{i}^{\mu}} \delta_{c^{1}, c_{i}^{\mu}}\left\{c \delta_{c_{i}^{\mu}, c_{i}^{\alpha}}+(1-c) \delta_{c_{i}^{\mu}, c_{i}^{\beta}}\right\}
$$

This gives at once

$$
\sum_{c^{\mu, \mu}} t_{\mu} c^{\mu} P\left(\begin{array}{l}
c^{\mu} \\
i
\end{array}\right)=c t_{\alpha}+(1-c) t_{\beta}=\bar{t}
$$

The independent averaging of a point can be denoted by a cross as in figure $2 \mathrm{~b}$. The next term in the sum (6) connects each repeat site with the previous occasion on which the site appeared and can be represented by a dotted line as in figure 2c. The third term contains a)

b)

c)

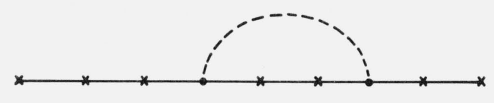

d)

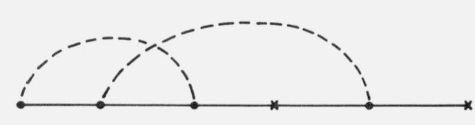

e)

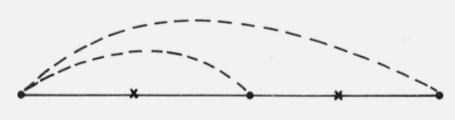

FIGURE 2. The diagrammatic expansion for the T-matrix showing (a) an unaveraged line, (b) the lowest order approximation, (c) a typical lowest order correction term, $(d)$ a second order correction term, and (e) a forbidden graph.

two repeats i.e. two dashed lines and so on. Indeed, the entire series (2) may be given by the diagrammatic expansion satisfying four simple rules. For each value of $\mathrm{n}, \mathrm{n}=1,2,3, \ldots$ draw all possible diagrams having the following properties

(i) There are $n$ points on a straight line.

(ii) Pairs of these points (but not a consecutive pair) are joined by dashed lines.

(iii) No two dashed lines leave a point in the same direction.

(iv) All points not touched by a dashed line have crosses on them.

The meaning of these diagrams can be readily put into words. For example figure $2 \mathrm{c}$ corresponds to a term in which the electron first scatters from 6 different sites then scatters again at the fourth one and finally scatters at two more different sites. Figure $2 d$ represents the electron returning to the first and second sites for its third and fifth scatterings. All such scattering topologies must be drawn and included in the series though it is important to note that diagrams of the type of figure $2 \mathrm{e}$ are forbidden by rule (iii). Finally, the dashed line does not represent the actual value of the process it 
describes but the correction to the related process in which the restriction is ignored and the two ends of the dashed line replaced by crosses. This is illustrated in the example below.

The evaluation of the contribution of a diagram is complicated by the two separate features involved. First there is the t-average which is given by the procedure outlined between eqs (3) and (6). Secondly the propagator sum must be evaluated for each diagram; it is this which is the limiting difficulty as the diagram becomes more and more complicated. Consider any diagram of the type $2 c$ i.e. with only one dashed line. The average corresponding to the dashed line may be written

$\sum_{c^{\mu}, c^{\nu}} t_{\mu} t_{\nu} \delta_{c^{\mu}, c_{i}^{\mu}} \delta_{c^{\nu}, c_{i}^{\nu}}\left\{c \delta_{c_{i}^{\mu}, c_{i}^{\alpha}}+(1-c) \delta_{c_{i}^{\mu}, c_{i}^{\beta}}\right\}$

$\times\left[\delta_{i j}-\left\{c \delta_{c_{j}^{\nu}, c_{j}^{\alpha}}+(1-c) \delta_{c_{j}^{r}, c_{j}^{\beta}}\right\}\right]=c t_{\alpha}^{2}+(1-c) t_{\beta}^{2}-\bar{t}^{2}$

$\delta_{i j}$ is written here as a reminder that $\mathrm{j}$ is exactly the same as $\mathrm{i}$ and was only introduced as a dummy variable. This is plainly of the form of the exact term less the zeroth approximation to it. Each cross on the line is replaced by $\bar{t}$ as in eq (7). Propagator lines which do not appear inside the dashed curve occur in the combination $\sum_{j(\neq i)} \mathrm{G}_{i j}=\mathrm{G}$. (In practice the sum is k-dependent;

$$
\boldsymbol{G}(\mathbf{k})=\sum_{j(\neq i)} G\left(\mathbf{R}_{i}-\mathbf{R}_{j}\right) e^{i \mathbf{k}\left(\mathbf{R}_{i}-\mathbf{R}_{j}\right)} .
$$

Between the ends of the dashed line the propagator must trace a scattering path beginning and ending at the same point so that the sum involved is

$$
S=\sum_{j(\neq i), k(\neq j) \text { etc. }} G_{i j} G_{j k} \ldots G_{m n} \delta_{n i}
$$

There is an additional restriction that no intermediate site can be that of the end point, otherwise rule (iii) will be violated and the correction terms overcounted. This sum is readily completed by Fourier transformation giving

$$
S=I /(1+t I)
$$

where

$$
I=\tau \int d \mathbf{k} G^{2}(\mathbf{k}) \bar{t}[1-\tau \bar{t} G(\mathbf{k})]^{-1}
$$

Here the appropriate number of $\bar{t}$ factors has been introduced and the integral in (9) is over the Brillouin zone of volume $\tau$.
The formal derivation above yields a diagrammatic expansion of a very familiar sort on which the usual tricks can be pulled. These will be discussed in the next section but it is appropriate that some feeling for the meaning of the diagrams be obtained. It will readily be seen that the expansion is not necessarily in powers of any small parameter of a physical nature (it might be e.g. if $\mathrm{t}$ were very small) but is more precisely viewed as an expansion in the disorder of the system. One way of looking at this is to consider the sequence of neighboring sites, i,j,k. Of the possible values of $k$, one will be $i$ and therefore ( 1 /number of values of $k$ ) of the terms will require corrections as discussed above. If $\mathrm{G}_{i j}$ is short ranged, as is the tight-binding case, this means that the proportion of correction terms is $1 / \mathrm{Z}$ (where $\mathrm{Z}$ is the number of near-neighbors) for each dashed line. An alternative is to regard the series as an expansion in the fluctuations of the system. While $1 / \mathrm{Z}$ appears to be small the number of terms with $\mathrm{n}$ dashed lines inceases something like $n$ ! as $n$ becomes large so that the series is at least asymptotically convergent. This is well known to occur in certain statistical mechanical expansions to which the above procedure is naturally related.

\section{The Simplest Approximations}

It is now possible to classify most of the theories of disordered alloys according to which diagrams they retain. This is useful in two ways. It gives information about the nature of the various approximations and it helps one to understand some of the physical parameters determining the convergence of the resummations. Begin with the simplest possible terms.

\subsection{The average $t$-matrix approximation [1]}

Ignoring all the diagrams with dashed curves gives at once the result

$$
\begin{aligned}
\langle T(\mathbf{k})\rangle & =\frac{N \bar{t}}{1-\bar{t} G(\mathbf{k})} \\
& =\frac{N}{\bar{t}^{-1}-(\mathbf{k})}
\end{aligned}
$$

in which the alloy is represented by a perfect lattice with identical scatterers having the average scattering of the alloy constituents. The density of states given by this approximation for a binary tight-binding alloy is shown in figure 3 . The tightly bound energy levels are $\mathrm{E}_{A}$ and $\mathrm{E}_{B}$ and the various cases are found by comparing $\left|\mathrm{E}_{A}-\mathrm{E}_{B}\right|$ with the bandwidth. While the gap between 


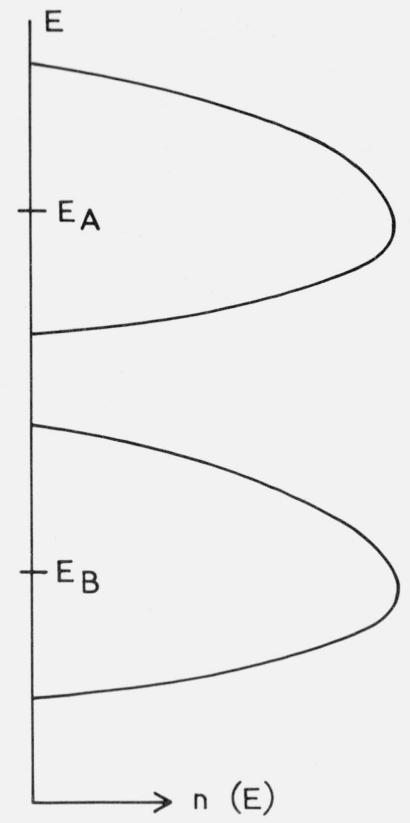

a)

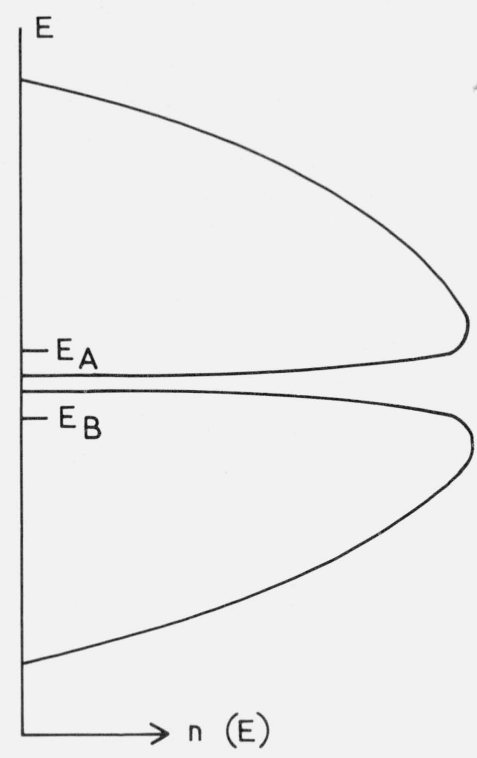

b)

FIGURE 3. The density of states in the average t-matrix approximation. (a) when $\left|\mathrm{E}_{A}-\mathrm{E}_{B}\right|>>$ bandwidth, (b) when $\left|\mathrm{E}_{A}-\mathrm{E}_{B}\right|<<$ bandwidth.

the band halves is reasonable when the bandwidth is much less than the energy splitting (fig. 3a), this approximation incorrectly predicts the gap even when the bandwidth is very large (fig. $3 \mathrm{~b}$ ). At the same time the rest of the band is moderately well described showing that the accuracy of the approximation for a given energy depends on the position of that energy within the band.

\subsection{First order approximation}

Under the assumption that the $1 / \mathrm{Z}$ series converges one might next evaluate the contribution of the terms of the type shown in figure 2c. This contribution must include all possible numbers of intermediate scatterings both inside and outside the dashed loop. But now consider the diagrams in figure $4 \mathrm{a}$. These each have an intermediate denominator which we expect to be nearly singular and all this sequence must be included too. This is simply the usual manipulation to give a self-energy rather than a t-matrix which is absolutely necessary in this context. Now

$$
T=\frac{N}{(\bar{t}+\Sigma)^{-1}-G}
$$

where $\Sigma$ is given by the diagram of figure $4 \mathrm{~b}$, with all possible numbers of intermediate states and can be evaluated from eqs (8) and (9). The inclusion of this term is an improvement over the average t-matrix approximation but does not resolve all the difficulties.
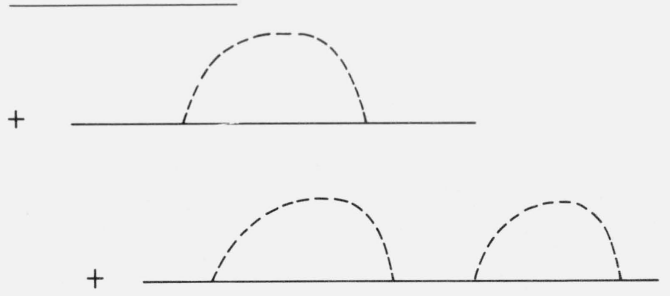

a)

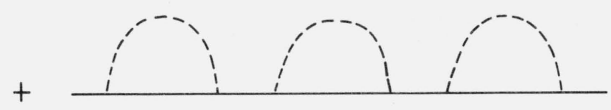

b)

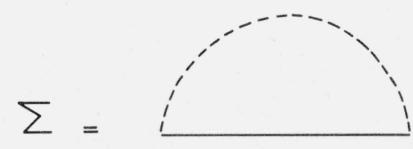

FIGURE 4. (a) the diagrams summed to give the self energy form, $(b)$ the definition of $\Sigma$. 

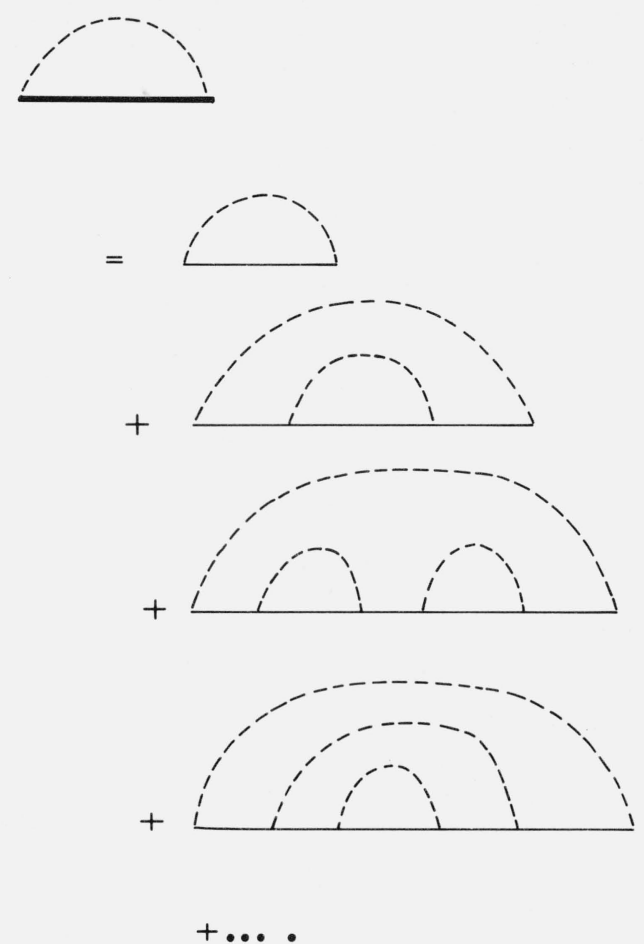

FigURE 5. The diagrams summed to give the self-consistent propagator inside the dashed line.

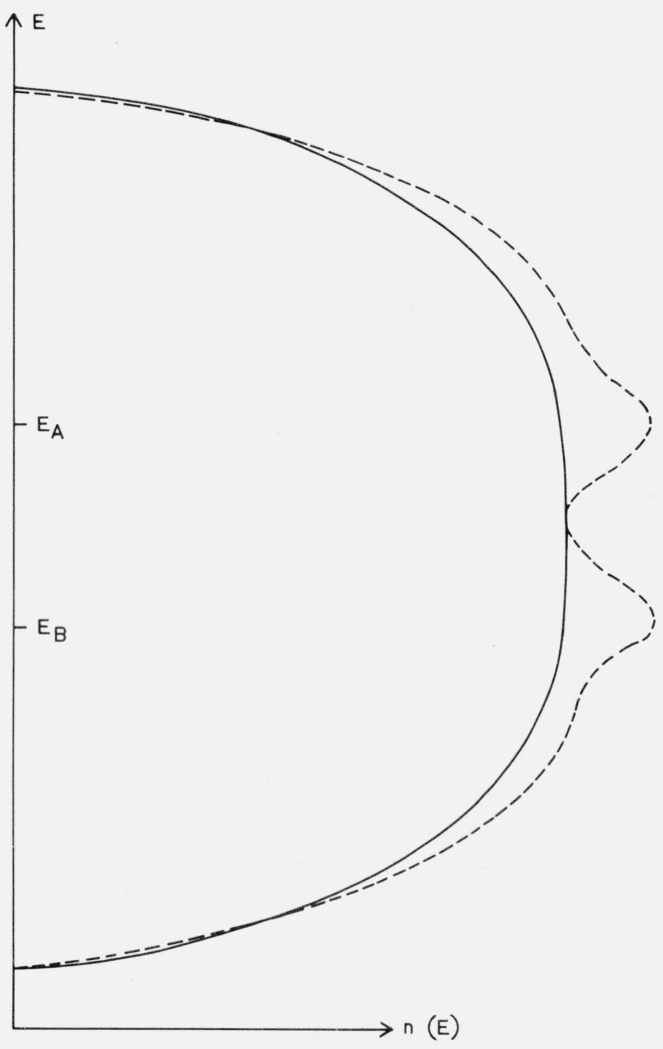

FIGURE 6. Some density of states curves showing approximations (iii) dashed lines and (iv) full iines for $c=0.5$ and $\left|\mathrm{E}_{A}-\mathrm{E}_{B}\right|=\Delta / 3.9$.

\subsection{Self-consistency}

It has long been known that using the unperturbed propagator in the expression for the self-energy is not the best that can be done. The same is true here, where the motion of the electron between successive scatterings can readily be included as if it were being scat tered by $(\bar{t}+\Sigma)$ scatterers. This corresponds to including the infinite series of diagrams seen in figure 5 . This step is necessary because one really wants to allow scattering of the electron into the true, not the unperturbed, density of states. Once this has been done the self-consistent equations obtained can be solved when the unperturbed density of states is given by the expression [2]

$$
\begin{aligned}
n_{o}(E) & =\frac{4}{\pi \Delta} \sqrt{1-\left(\frac{E-T_{o}}{\frac{1}{2} \Delta}\right)^{2}} \text { if }\left|E-T_{o}\right|<\frac{1}{2} \Delta \\
& =0 \quad \text { otherwise. }
\end{aligned}
$$

This self-consistency calculation is an immediate improvement in the sense that the band gap now closes as the splitting $\left|\mathrm{E}_{A}-\mathrm{E}_{B}\right|$ becomes smaller than the bandwidth. Figures 6 and 7 show the alloy density of states in

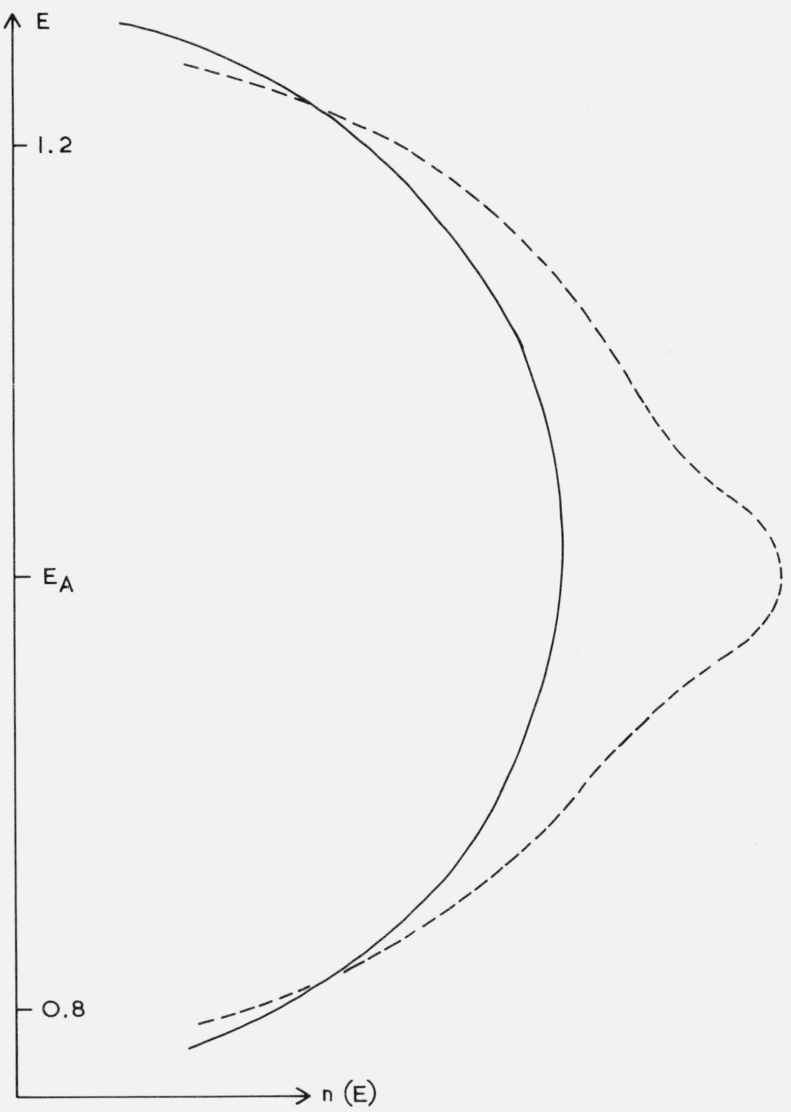

FigURE 7. The density of states curves for $c=0.5$ and $\left|\mathrm{E}_{A}-\mathrm{E}_{B}\right|=\Delta / 0.7$. The scale here is such that $\mathrm{E}_{A}=+1, \mathrm{E}_{B}=-1.0$. 

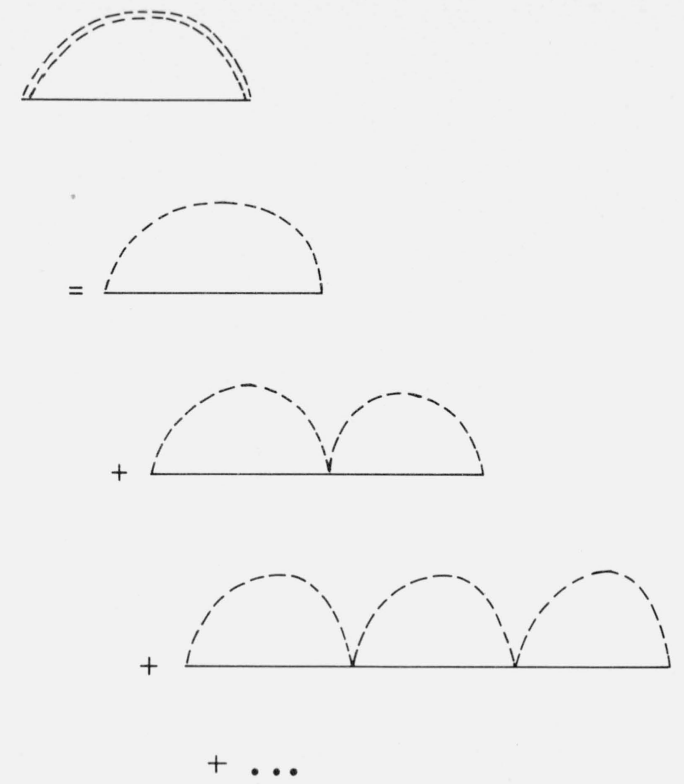

FIGURE 8. The self-energy graphs required to renormalize the scattering energy levels.

the approximation as a dashed line. One major error of such a density of states is that it does not satisfy the sum rule on the density of states, a difficulty that can be overcome by a further resummation.

\subsection{The renormalized energy levels}

The work of Hubbard [2] and later workers [3,4] overcomes the sum rule problem by extending the scattering from any single site to include all possible processes involving only that site. These are the diagrams of figure 8 . This has the effect of renormalizing the energy levels and gives the full line density of states in figures 6 and 7. It will also be noticed that the bump in $\mathrm{n}(\mathrm{E})$ near $\mathrm{E}_{A}$ and $\mathrm{E}_{B}$ has flattened out. Thus overall this more sophisticated summation gives a smoother density of states. It is interesting to observe that neither the value of the gap width nor the critical ratio $\left|\frac{E_{A}-E_{B}}{\triangle}\right|$ at which the gap closes is affected by this last improvement. Within the numerical error approximations (6.3) and (6.4) are identical in this respect.

This last approximation has allowed several alloy density of states calculations to be carried through $[3,4]$ and is probably the best that can be done at the moment. It is rather difficult to see what to do next. There is no general rule for selecting sets of diagrams obeying the density of states sum rule and one has no insight into which of the higher order terms are the most important. Indeed different sets will probably be important in different regions of the energy spectrum.

\section{Dimensions and Fluctuations}

This section is intended to draw attention to a few of the difficulties associated with the alloy theories just outlined. Rather than dealing directly with the alloy case it is preferable to simplify to the vacancy problem. Here the second alloy species is replaced by vacancies so that only a proportion $\mathrm{c}$ of the perfect lattice sites are occupied by type A atoms. In the formalism of the preceding sections it is only necessary to put $\mathrm{t}_{\beta} \equiv 0$.

The illustrative value of the number of dimensions in this argument arises because the formed procedure just outlined is independent of the dimensionality for fixed unperturbed $n(E)$. The physical nature of the problem, however, depends extremely strongly on the dimension principally through the importance of fluctuations. $\mathrm{Ob}$ viously the fluctuations in one dimension are very large compared to those in three dimensions since the number of neighbors is so much less. This is reflected in the fact that in one dimension the density of states for the alloy problem has a strongly peaked structure, the peaks being identifiable as local groupings of a few atoms as was first remarked upon by Borland [5]. In particular, for the vacancy tight binding cases, the probability of finding a line of $n$ occupied sites with a vacancy on either end is $c^{n}(1-c)^{2}$ and the density of states for such a group is $\sum_{i=1}^{n} \delta\left(\mathbf{E}-\mathbf{E}_{i}\right)$. The total density of states is then effectively a weighted sum over $\delta$ functions and is not continuous. Obviously in two and three dimensions the same is true for sufficiently small concentrations but not in general. Plainly when fluctuations are this important there is no chance that the theory described previously can hold.

Another point which can be readily demonstrated is that in such a vacancy case there exist states outside the bands calculated by any of the models just discussed. Consider a simple square lattice in two dimensions. This has coordination 4. A square of side $\mathrm{n}$ fully occupied by atoms will have a mean coordination number

$$
\frac{4 n^{2}-4 n}{4 n^{2}}=1-\frac{1}{n}
$$

since atoms at the sides only have coordination 3 . In a tight binding model the mean coordination number is a least estimate for the bandwidth of a group of atoms compared to that for the full lattice. This can be readily seen by a variational calculation with trial function $\Psi$ $=\sum_{i=1}^{n^{2}} \Psi_{i} / \mathrm{n}$. Now the theories described above give a bandwidth $\propto$ (approx. (6.1)) or $\sqrt{c}$ (approx. (6.4)) so for 
the latter case there is a probability $c^{n^{2}} \simeq c^{(1-\sqrt{c})-2}$ of groups of atoms with energy levels outside the calculated band. Such states of course tail off rapidly but nevertheless exist with positive density of states right out to the full perfect lattice bandwidth. The same applies in three dimensions where the tail is smaller but still there. Figure 9 illustrates this point. Obviously such states are very difficult to spot in the type of theory discussed earlier. They certainly exist at the outside edges of the alloy bands but whether or not they exist inside the band gap is a more difficult question which will undoubtedly be the object of more study.

\section{The Experimental Comparison}

It is natural enough that when developing a theory of alloys one begins with the simpler, one-body quantities such as the density of states. Other properties, such as conductivity, Hall effect, etc. really require more sophisticated theoretical treatments. The relevant experiments are well dealt with elsewhere in the symposium and so will not be discussed in detail here, but it would be wrong to completely omit commentary on them.

Calculations of the density of states yield a function of energy and a proper experimental comparison would require that function to be observed. Only in the optical type measurements, particularly photoemission and soft $\mathrm{x}$-ray work, is this possible even in principle. In practice there is a good deal of ambiguity in separating out the $n(E)$ curves from other energy dependent variations typically in the matrix elements and many-electron effects. It is clear that for a general comparison with experiment the theoretical work must be pushed to the point of predicting directly the observed data. This is a step which at the moment looks to be just about possible though complicated by the need to take into account local effects through the matrix elements.

Those experiments which measure densities of states at the Fermi surface are complicated by many-body features which, for example, can cause the density of states to be enhanced by quite large factors $(\sim 1.5$ or more). There is the additional difficulty that the pure transition metal band structures lead to density of states curves with a good deal of structure on them so that detailed comparison with experiment requires precise knowledge of potentials and the Fermi energy. Beck and coworkers [6] have overcome some of these objections by using transition metal alloys which seem to conform very closely to the rigid band model and in which the parameters vary only with electron concentration. It is still questionable whether theory can yet

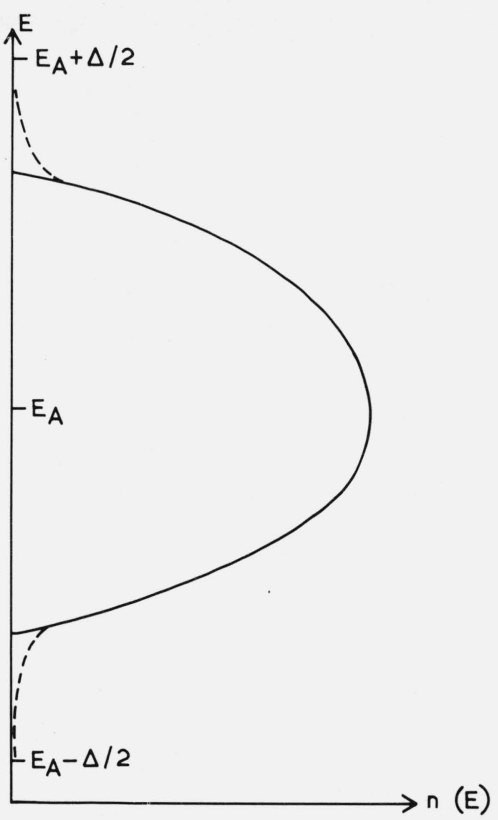

FIGURE 9. Density of states in the vacancy case showing the limits of the perfect band and the tails extending to those limits.

predict those cases where Beck's attack will work and in particular where and why it breaks down in the transition metal alloys.

The need for an indirect step in all these experimental analyses has hitherto prevented a satisfactory comparison between the calculated and the measured density of states. It now seems possible that since sophisticated yet fast band structure calculations have been developed and better theoretical understanding of the alloy problem is being rapidly gained the time should soon come when the comparison will become direct.

Finally one might expect some rewards by looking for the gap predicted by the theories when the energy levels are well split as in figure $3 \mathrm{a}$. However, this particular gap is strongly reminiscent of those predicted in the more general disordered system theories and still argued about at length. It is very difficult to observe the difference between a gap and a very low flat minimum even though the distinction is theoretically very important. Perhaps the tunnelling experiments can help here.

\section{Conclusions}

The theory of disordered alloys is at last coming to the point where it can truly be regarded as a theory rather than being a collection of ad hoc methods. The 
problem of a disordered alloy can thus be treated in a parallel fashion to many-body theories and in consequence considerable experience taken over from the many-body theorists. At the same time there remains a real difficulty in producing a satisfactory dialogue with the relevant experiment work. Overall, however, the picture is one holding promise of good developments in the near future which may have a catalyzing effect on the theoretical understanding of disordered systems.

\section{References}

[1] Beeby, J. L., Phys. Rev. 135, Al30 (1964).

[2] Hubbard, J., Proc. Roy. Soc. A281, 401 (1964).

[3] Soven, P.,Phys. Rev. 156, 809 (1967); Onodera, Y. and Toyozawa, Y., J. Phys. Soc. Japan 24, 341 (1968).

[4] Velicky, B., Kirkpatrick, S., and Ehrenreich, H., Phys. Rev. 175, 747 (1968).

[5] Borland, R. E. and Agacy, R. L., Proc. Phys. Soc. 84, 1017 (1964).

[6] See for example, Cheng, C. H., Gupta, K. P., van Reuth, E. C., and Beck, P. A., Phys. Rev. 126, 2030 (1962).

(Paper 74A2-600) 